\title{
Virtual pre-operative planning in acetabular surgery using a patient-specific biomechanical model: a prospective clinical study.
}

\author{
Mehdi Boudissa $^{1,2}$, Matthieu Chabanas ${ }^{2}$, Gaetan Bahl' ${ }^{2}$ Hadrien Oliveri² \\ and Jérôme Tonetti ${ }^{1,2}$ \\ ${ }^{1}$ Orthopedic and Traumatology Surgery Department, Grenoble University Hospitals, La Tronche, \\ 38700, France \\ ${ }^{2}$ TIMC-IMAG lab, Univ. Grenoble Alpes, CNRS UMR 5525, La Tronche, 38700, France \\ mboudissa@chu-grenoble.fr
}

\begin{abstract}
The first patient-specific biomechanical model for pre-operative planning in acetabular surgery was developed in our institution and validated in previous retrospective studies. The aim of this prospective clinical study was to confirm the previous promising.

Between January 2019 and June 2019, every patients operated by the first author for acetabular fracture were included in this prospective study. A biomechanical model was implemented in acustom software made from combination of several open-sources software allowing a biomechanical simulation. The surgery was then performed according to the simulation. Surgery duration, blood loss, radiological results and peroperative complications were recorded.

Ten patients were included. Mean simulation time was $22 \min \pm 4$ [range, 18-31]. The mean operative time was $113 \mathrm{~min} \pm 33$ [range, 60-180] and mean blood loss was $505 \mathrm{~mL}$ \pm 189 [range, 100-750]. On Matta's criteria, anatomic reduction was achieved in 9 of the 10 patients $(90 \% ; 0.8 \mathrm{~mm} \pm 1$ [range, $0-3])$. No per-operative complications were recorded.

This study confirms the promising results of pre-operative planning in acetabular surgery based on a patient-specific biomechanical model.The model needs larger-scale prospective validation, but offers a new tool suitable for teaching purposes and for assessment of surgical strategies in acetabular fracture.
\end{abstract}




\section{Introduction}

Several tools for preoperative planning in acetabular fracture have been presented in the literature Studies report improved understanding and definition of surgical strategy, with operative time-saving, reduced bleeding and improved reduction [1].

All these preoperative planning tools are geometrical repositioning with their own limitations. Indeed, reducing the fracture in 3D through mouse interactions is difficult, quite non-intuitive and hardly guaranty non-penetration between fragments. An intuitive simulation of the fracture reduction using a mechanical model was developed with promising results [2]. To our knowledge, it is the first biomechanical model used in daily clinical routine for virtual planning in acetabular surgery.

This prospective clinical study intends to confirm the previous promising results.

\section{Materials and Methods}

Between January 2019 and June 2019, patients operated by the first author for acetabular fracture were included.

A 3D model of the bone fragments was constructed by semi-automatic segmentation using the open-source ITK-Snap package (ITK-Snap, Philadelphia, PA). After importation of DICOM images from the pre-operative high-resolution CT scans, the region of interest was selected by thresholding bone density so as to segment only bone structures including fractured fragments. The software then performed segmentation automatically by a region growing algorithm. Manual refinement was sometimes needed to separate the various fragments in comminuted fractures [3].

A patient-specific biomechanical model was constructed and implemented in the open-source ArtiSynth (ArtiSynth, Vancouver, Canada) simulation environment [4]. Each bone fragment was considered as an independent rigid body. Bone attached to the sacro-iliac joint was considered to be fixed, whereas the other fragments were considered mobile. Collisions were considered, to ensure against bone fragment interpenetration. The action of the various surgical instruments (forceps, Schanz screws, traction, etc.) was simulated in translation and rotation. To make the simulation realistic, the muscles and their effective action on the bone were simulated by applying global damping on the system, corresponding to a field of viscosity simulating the action of curarized muscles. This simple but effective "trick" avoided any non-anatomic displacement or numerical instability relating to the forces applied on the fragments. The tissues involved in the reduction and balancing of the system (i.e., joint capsule, sacrospinous and sacrotuberous ligaments, inguinal ligament and pubic symphysis) were modeled and set in the model by the surgeon according to the type of fracture [2]. The system does not suggest anything. It uses forces \& damping between the fragments and the surgeon positions them using the mouse. The soft tissue properties are generic according to the literature. Each patient-specific model was constructed by the surgeon and adapted to the type of fracture (figure 1).

After simulation, the surgery was performed. Operative time and blood loss were recorded. The peroperative complications were also recorded. Reduction quality was assessed on a postoperative CT scan by an independent observer using Matta's criteria [5]. 


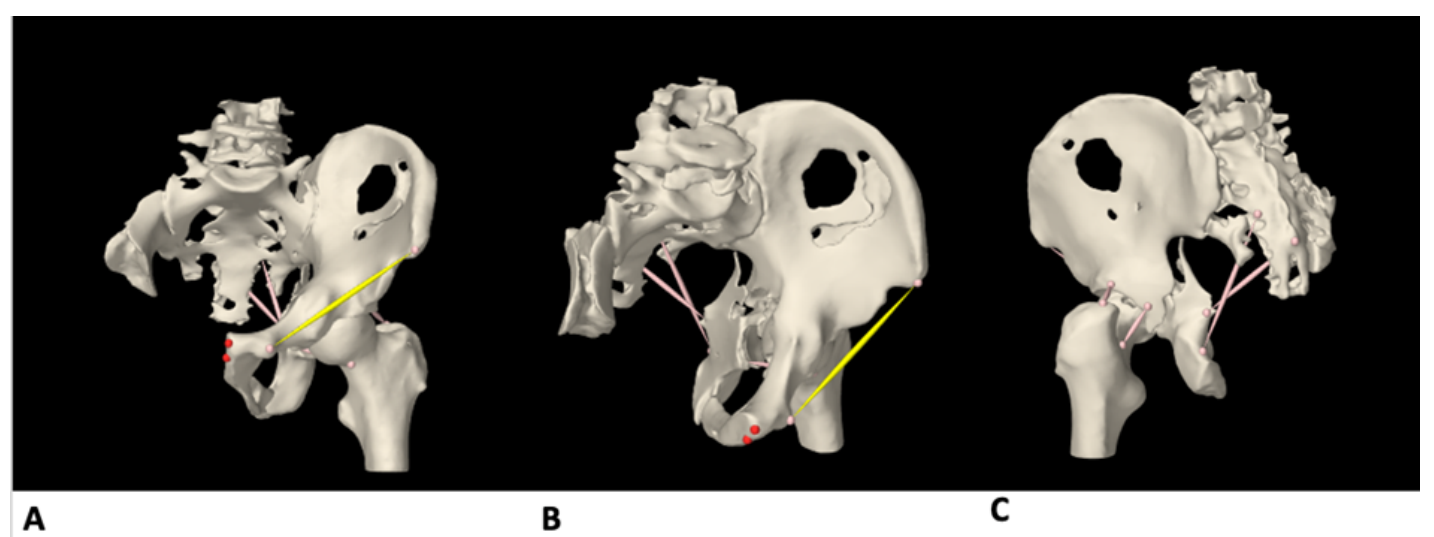

Figure1: Biomechanical model with modeled soft tissues: joint capsule (pink), sacrospinous and sacrotuberous ligaments (pink), inguinal ligament (yellow), and pubic symphysis (red) with anteroposterior view (A), endopelvic view (B) and exopelvic view (C).

Statistical analysis used StatView 5.5 software (SAS Institute, Cary, NC, USA).

\section{Results}

Ten simulations and 10 surgeries were performed. The same reduction sequences as previously simulated were reproduced during surgery, and reduction quality was compared. Biomechanical simulation systematically reproduced the bone fragment behavior observed intraoperatively, and the reduction quality. Anatomic reduction was achieved in 9 patients $(90 \% ; 0.8 \mathrm{~mm} \pm 1[0-3])$ and in the 9 corresponding simulations $(90 \% ; 0.5 \mathrm{~mm} \pm 0.9[0-3]), \mathrm{p}=0.53$. The mean operative time was $113 \mathrm{~min}$ \pm 33 [range, 60-180] and mean blood loss was $505 \mathrm{~mL} \pm 189$ [range, 100-750]. Results are shown in Table 1. Mean semi-automatic segmentation time was $82 \min \pm 18$ [range, 60-120]. Mean simulation time was $22 \min \pm 4$ [range, 18-31]. 


\begin{tabular}{|c|c|c|c|c|c|c|}
\hline case & $\begin{array}{l}\text { Age (years) / } \\
\text { Gender }\end{array}$ & $\begin{array}{l}\text { Fracture patterns } \\
\text { (Letournel } \\
\text { classification) }\end{array}$ & $\begin{array}{l}\text { Time of simulation } \\
\text { (excluding } \\
\text { segmentation)(min) }\end{array}$ & Surgical approach & $\begin{array}{c}\text { Reduction } \\
\text { quality } \\
\text { according to } \\
\text { Matta [12] }\end{array}$ & $\begin{array}{c}\text { Virtual planning } \\
\text { correlation }\end{array}$ \\
\hline 1 & 27 / Male & $\begin{array}{c}\text { Transverse + posterior } \\
\text { wall } \\
\end{array}$ & 20 & Kocher-Langenbeck & anatomical & Identical \\
\hline 2 & 55 / Male & $\begin{array}{c}\text { Anterior column + } \\
\text { posterior } \\
\text { hemitransverse }\end{array}$ & 18 & Stoppa & imperfect & Identical \\
\hline 3 & $61 /$ Male & T type & 25 & Stoppa & anatomical & Identical \\
\hline 4 & 29 / Male & Both column & 18 & Ilio-inguinal & anatomical & Identical \\
\hline 5 & 86 / Male & Anterior column & 19 & Keel & anatomical & Identical \\
\hline 6 & $50 /$ Male & $\begin{array}{l}\text { Anterior column + } \\
\text { posterior } \\
\text { hemitransverse }\end{array}$ & 25 & Stoppa & anatomical & identical \\
\hline 7 & $51 /$ Male & $\begin{array}{c}\text { Anterior column + } \\
\text { posterior } \\
\text { hemitransverse }\end{array}$ & 31 & Stoppa & anatomical & Identical \\
\hline 8 & $86 /$ Male & $\begin{array}{c}\text { Anterior column + } \\
\text { posterior } \\
\text { hemitransverse }\end{array}$ & 19 & Stoppa & anatomical & Identical \\
\hline 9 & 67 / Male & Anterior column & 20 & Ilio-inguinal & anatomical & Identical \\
\hline 10 & $41 /$ Male & Both column & 24 & $\begin{array}{c}\text { Stoppa + first window } \\
\text { of ilio-inguinal } \\
\text { approach }\end{array}$ & anatomical & Identical \\
\hline
\end{tabular}

Table 1: Comparison between biomechanical simulations and surgeries.

\section{DISCUSSION}

The main interest of this preoperative planning tool derives from the new paradigm according to which it is the actual procedure rather than the desired result that is to be simulated [2]. In the literature, all authors describe geometric models for repositioning each bone fragment by moving them freely in 3 dimensions, in translation and rotation [6-8]. In a previous study we had reported the limitations of these planning. Indeed, this does not match surgical reality: fragments cannot always be considered independent, as they are sometimes connected by elements such as the joint capsule or sacrospinous ligament. The present biomechanical model corresponds to a stable numeric environment comprising bone fragments, soft tissue and their interactions [2]. A retrospective study had shown that this patientspecific biomechanical model faithfully reproduces bone fragment behavior with respect to the surgical reduction sequences [2]. The present prospective study confirms the previous promising results in a daily clinical routine.

The model needs larger-scale prospective validation, but offers a new tool suitable for teaching purposes and for assessment of surgical strategies in acetabular fracture. To our knowledge, this is the first patient-specific biomechanical simulator of acetabular fracture reduction. 


\section{References:}

[1] Boudissa M, Courvoisier A, Chabanas M, Tonetti J. Computer assisted surgery in preoperative planning of acetabular fracture surgery: state of the art. Expert Rev Med Devices 2018;15:81-89.

[2] Boudissa M, Oliveri H, Chabanas M, Tonetti J. Computer-assisted surgery in acetabular fractures: Virtual reduction of acetabular fracture using the first patient-specific biomechanical model simulator. Orthop Traumatol Surg Res 2018;104:359-362.

[3] Yushkevich PA, Piven J, Hazlett HC, Smith RG, Ho S, Gee JC et al. User-guided 3D active contour segmentation of anatomical structures: Significantly improved efficiency and reliability. Neuroimage 2006;31:1116-28

[4] Loyd J, Stavness I, Fels S.Springer, editor. ArtiSynth: A fast interactive biomechanical modeling toolkit combining multibody and finite element simulation. Soft Tissue Biomechanical Modeling for Computer Assisted Surgery; 2012, p. 355-394.

[5] Matta JM, Merritt PO. Displaced acetabular fractures, Clin Orthop Relat Res 1988;230:83-97.

[6] Cimerman M, Kristan A. Preoperative planning in pelvic and acetabular surgery: The value of advanced computerised planning modules. Injury 2007;38:442-49.

[7] Hu Y, Li H, Qiao G, Liu H, Ji A, Ye F. Computer-assisted virtual surgical procedure for acetabular fractures based on real CT data. Injury 2011;42:1121-24.

[8] Fornaro J, Keel M, Harders M, Marincek B, Székely G, Frauenfelder T. An interactive surgical planning tool for acetabular fractures: initial results. J Orthop Surg Res 2010;4:5-50.

Acknowledgments: The study received support from ANR France and the Fondation pour l'Avenir (labex CAMI), reference ANR-11-LABX-0004. 